\title{
FARADAY LECTURE.
}

(Delivered before the Fellows of the Chemical Society in the Theatre of the Royal Institution on Friday, October 18th, 1907.)

\section{Synthetical Chemistry in its Relation to Biology.}

\section{By Emil Fischer, F.R.S.}

Exactin forty years are elapsed since Faraday's death and already, on eight occasions, discourses in honour of his memory have been delivered, at the instance of this Society, by distinguished representatives of the sciences which he did so much to forward. Most of them, mindful of Faraday's discoveries, have dealt with general problems connected with our fundamental conceptions of matter and its inherent forces, so that, when honoured with the invitation to deliver the ninth of these lectures, I could not but feel sensible of the difficulty of following the example of my predecessors, as the field in which I have sought to enlarge the boundaries of knowledge is one far removed from such considerations.

In Faraday's time, it is true, the labours of workers in the natural sciences were not specialised as they are now; his genius was such, however, that he was able, although not trained in its service, to enrich organic chemistry with observations of momentous consequence. I need but allude to his discovery of benzene: the practical importance of this substance and its dominant position in the host of carbon compounds with which we are acquainted were generally recognised in the course of the celebration held in this room, only fifteen months ago, in honour of Sir William Perkin.

But in the course of his labours as an investigator Faraday never approached the attractive problems which organic chemistry affords on its biological side. I must therefore crave your indulgence in selecting this theme in particular as the subject of my address. Happily, I am sustained by the conviction that biology, on account of the breadth and importance of its aims, may claim to rank with the inorganic sciences, although perhaps it lacks their exactness of method; nowhere can it count on fuller recognition of its achievements and of the importance of its problems than among the countrymen of Charles Darwin.

It is easy to understand why in its early youth organic chemistry was so closely connected with biology; the materials which the chemist was called upon to investigate were mostly products of animal or vegetable origin. Indeed, carbohydrates, proteins and 
vegetable acids served Lavoisier, Gay-Lussac, Berzelius and Liebig as materials in elaborating the methods of elementary analysis.

The isolation of urea from animal urine by Rouelle, the recognition of uric acid, lactic acid, malic acid and glycerine by Scheele, the isolation of asparagine by Vauquelin and Robiquet, of morphine by Serturnier, together with many other similar discoveries accomplished during the first ten years of the nineteenth century, are admirable examples of the manner in which the living world was drawn upon and made to yield up its treasure of chemical compounds. The many hundreds of natural organic compounds enumerated in the text-books of animal and vegetable chemistry are proof of the rich harvest since gathered in this field of investigation. But how small is their number when compared with the 130,000 carbon compounds which organic chemistry can boast of to-day. All these, it is known, are either products of the artificial transformation of organic matters occurring naturally or have been completely synthesised from their elements. The accumulation of this huge material, including the elaboration of the necessary methods, has been the main occupation of organic chemists during the past sixty years; and as their discoveries gave rise to much happy speculation, they often took the lead in developing chemical theory.

It is not to be denied that, in the latter half of the last century, owing to the growth of the subject in importance, organic chemistry became separated from biology. It cannot be mere chance that the most famous of Liebig's pupils, A. W. Hofmann, A. Kekulé and A. Wurtz, did not follow the example of their great teacher, whose chief triumphs were won by the use he made of chemical methods in solving biological problems. Perhaps they were restrained by the feeling that, mainly through his influence, physiological chemistry had been developed into a separate discipline, which should be cared for by men who could devote themselves entirely to its service. Such subdivision of labour undoubtedly has many advantages; the disadvantages would have outweighed these had it precluded interchange of experiences and friendly co-operation of workers in the two fields; the history of both sciences, however, affords ample proof that such has not been the case.

Physiologists have ever been ready to avail themselves of the latest developments of chemical analysis and synthesis, whilst organic chemists have not only been stimulated in many ways by biologists but their studies have derived much practical aid from biological science. I may instance the modern development of the chemistry of fermentation, which began with the pioneer 
work of Pasteur and was greatly favoured by the introduction of refined bacteriological methods by Koch and Hansen; also the flourishing industry to which the manufacture of medical remedies prepared by synthetic methods has given rise.

But organic chemistry will certainly never be content to act as the mere handmaid of biology. This is impossible, as the theoretical and technical problems which she is called upon to consider are already numerous and they cannot fail to increase in number and importance in the future. But I do consider it not only possible but desirable, that the close connexion of chemistry with biology which prevailed in the days of Liebig and Dumas should be re-established, as the great chemical secrets of life are only to be unveiled by co-operative work. I will therefore attempt to indicate the part chemistry can play by reference to cases of which I can claim to have personal experience.

We know that in nature the construction of organic matter begins in the leaves of plants with the conversion of carbon dioxide into sugar, from which many physiologists suppose the complex substances contained in the living cell are formed by further changes in which nitrogen, sulphur and phosphorus take part.

These transformations are for the most part enveloped in mystery. We know nothing definitely even of the assimilation of carbon dioxide. Of the various hypotheses advanced to explain the change, that advocated by $\mathrm{A}$. von Baeyer has gained most support, namely, the view that the initial product is formaldehyde, glucose being formed from this by a process of polymerisation. Actually both changes have been effected by artificial means. After it had been shown by Butleroff that a sugar-like, syrupy product is formed on heating formaldehyde with lime water and $\mathrm{O}$. Loew had improved the method of effecting the condensation, I was able to adduce proof that the complex mixture contains a small quantity of a-acrose which can be transformed into glucose. The preparation of glucose from carbon dioxide thus became a possibility, as it was known that carbon dioxide could be converted into formaldehyde by more or less drastic processes. Recently, Fenton has succeeded in carrying out the reduction of carbon dioxide to formaldehyde at a low temperature in aqueous solution, so that it is now possible to effect the complete synthesis of sugar at temperatures such as prevail in the living plant. But how thorough is the work of the plant in comparison with our laboratory practice; usually when such questions are discussed, the poor yields which our methods give rise to are forgotten!

I need only allude here to recent apparently successful attempts, on the one hand, to effect the reduction of carbonic acid to form- 
aldehyde by means of light and, on the other, to detect formaldehyde in green leaves, as Professor Meldola dealt exhaustively and critically with these questions in his Presidential Address eighteen months ago. I may be allowed, however, to dwell somewhat on one peculiar feature of the natural change, namely, the asymmetric character of the synthesis; according to present knowledge-I may refer especially to the brilliant investigations of $\mathrm{H}$. Brown and Morristhe optically active hexoses of the $d$-series, glucose and fructose, are alone formed.

But from the experience gained in effecting syntheses in the sugar group, as I showed some time ago, it is possible to give a fairly satisfactory explanation of this change. It is only necessary to assume that the condensation is preceded by the formation of an additive compound of formaldehyde with some optically active constituent of the chlorophyll granules. I shall give a more precise form to this hypothesis if I say that I consider it probable that the carbon dioxide itself enters into combination in this manner, as there is reason to suppose that the proteins offer sufficient opportunity for its fixation; according to Siegfried, even the simple aminoacids are capable of combining with carbon dioxide. I am inclined to think that this compound with carbon dioxide undergoes decomposition into oxygen and a reduction product, probably a derivative of formaldehyde; the condensation to sugar takes place either in the original asymmetric complex or in one produced from it by a secondary change involving the separation of the formaldehyde and its re-association in some other manner. It may be that the condensation takes place directly or that intermediate compounds, biose or glycerose, are formed. Thanks to the researches of Marckwald and especially those of Mackenzie, we are acquainted with a whole series of asymmetric syntheses; no one of these, however, is half so complete as that involved in the formation of sugar under natural conditions. Indeed, it is obvious that if the natural process is to be imitated in vtro, it will be necessary to alter the methods hitherto adopted in every single detail; difficult as this may appear, it is not altogether impossible.

But even if this be done successfully, the precise nature of the assimilation process will not be finally elucidated. It is to be expected that this will only be accomplished when biological research, aided by improved analytical methods, has succeeded in following the changes which take place in the actual chlorophyll granules.

The carbohydrates elaborated by the plant undergo combustion to carbon dioxide and water in the animal body. The change is easily effected by means of powerful oxidising agents at the ordinary temperature; the natural process, however, must be a very different 
one, as in the organism oxygen is oonveyed to the carbohydrate by oxidising enzymes and doubtless many intermediate products are formed of which we know little at present.

It would be easy to multiply examples. But these two are sufficient to demonstrate the incompleteness of the explanation of biochemical processes deduced from the data of organic chemistry. The service rendered to biology by chemical analysis and synthesiswhich will be rendered to it in even greater measure in the futureis to be sought in other directions.

The ultimate aim of biochemistry is to gain complete insight into the unending series of changes which attend plant and animal metabolism. To accomplish a task of such magnitude, complete knowledge is required of each individual chemical substance occurring in the cycle of changes and of analytical methods which will permit of its recognition under conditions such as exist in the living organism. As a matter of course, it is the office of organic chemistry, especially of synthetic chemistry, to accumulate this absolutely essential material. The chemical constitution of hundreds of carbon compounds which occur naturally has already been determined and their more important properties have been established. But far more remains to be done. In proof of this, let me briefly direct your attention to the three great classes of substances which predominate in the living world: the fats, the carbohydrates and the proteins.

It was established at least ninety years ago by Chevreul, in the course of his celebrated investigations into the process of soapmaking, that the fats can be decomposed into the glycerine discovered by Scheele and into fatty acids; but the relationship of these latter to one another could not be understood until the conception of homologous series had been evolved in organic chemistry. The classical researches of Berthelot and the discovery of glycol by Wurtz were necessary preliminaries to the establishment of the constitution of glycerine; the final proof that the fats are neutral glyceric salts of the fatty acids was first provided by Berthelot's synthesis. Synthetic methods have made us acquainted with the mono- and di-glycerides and also with mixed triglycerides such as have frequently been met with of late in nature.

Nevertheless, the group in which the natural fats are ranged is one in which there are still many gaps and many misstatements to be corrected. Fatty acids of high molecular weight, which for years were regarded as entities, such as margaric acid, are now acknowledged to be mixtures; others, like the cerotic acid of beeswax, have received new formulæ; in fact, the higher acids and the oxy-acids all require to be studied afresh and, whilst it is true that 
the general constitution of the fats is known with certainty and the quantitative composition of the most important natural mixtures is established with a fairly satisfactory degree of accuracy, yet there is much uncertainty on the physiological side; even such an apparently simple problem as that involved in the determination of the manner in which fats are absorbed in the animal intestine has not yet been definitely solved. Although enzymes capable of cleaving fats are known to be present in the secretions of the stomach and pancreas, opinions are still divided as to whether the fat is absorbed in a state of fine emulsion or whether it first undergoes bydrolysis.

Still less is known of the manner in which fats undergo combustion to carbon dioxide and water in the animal body. Hitherto this change has been imitated artificially only at relatively high temperatures. It is obvious that it would be of far greater interest to discover oxidising agents which would produce the effect at low temperatures. No doubt many intermediate products would be met with and the study of these would afford valuable assistance to biologists in devising experiments with animals.

The formation of fats in plant and animal tissue is no less complicated a phenomenon. It is certain that carbohydrates are the chief source of supply. We know next to nothing, however, of the actual course of this remarkable synthesis; it is easy to imagine that glycerol may be formed by the hydrolysis of glucose and that three molecules of glucose may give rise to an acid containing eighteen carbon atoms. But the process involved in the conversion of sugar which is rich in oxygen into fatty acids containing little oxygen remains wrapped in mystery. I imagine that this change, like that which attends alcoholic fermentation, is effected by a rearrangement of oxygen atoms within the molecule and that it involves the elimination of carbon dioxide. To imitate such a process artificially, of course, is an attractive problem to be dealt with from the synthetic side; but it is doubtful if it can ever be solved by purely chemical methods. In all probability, the organism has at its disposal for the purpose a number of enzymes which effect the complete synthesis in a series of interactions following rapidly one upon another.

The problems afforded by the fats are simple, however, in comparison with those connected with the carbohydrates. The original subdivision of the group into mono-, di-, tri- and poly-saccharides has been justified in practice. $U p$ to the present time only the monosaccharides have been studied satisfactorily from the point of view of their spatial structure. The dogma which at one time 
prevailed that Nature produces only carbohydrates with six atoms of carbon was upset by Kiliani's discovery that arabinose contains but five atoms of carbon. Since then the group of the monosaccharides has been so extended by synthetic means that sugars of all types, from glycollaldehyde (biose) to the nonoses, are now known, the group including besides the seven compounds which occur naturally no less than forty-five synthesised products.

Our knowledge of this group is also fairly satisfactory from the stereochemical standpoint, as the greater number of the isomerides predicted by theory as well as the more important aldohexoses and aldopentoses have been prepared and their inter-relationship can be expressed by steric formulæ. Similar results have been arrived at in the case of the great class of glucosides, the synthesis of the most simple representative from glucose and methyl alcohol having led to the discovery of both the theoretically possible stereoisomerides.

The growth of our knowledge of the monosaccharides has proved in many ways to be of importance in connexion with biological inquiry, especially in enabling us to penetrate the mystery of enzyme action somewhat further.

On contrasting the effects which emulsin and the enzymes in yeast produce on the various glucosides prepared by synthetic methods, I was led to conclude not only that there was a difference between the two series of optical antipodes similar to that discovered by Pasteur in the course of his studies of moulds but that very slight changes in configuration were sufficient to inhibit the action of enzymes entirely. I was led by these observations to apply the simile of lock and key as an expression of the close inter-relationship in configuration which obtains between the enzyme and the substance which it attacks.

Similar results were obtained on investigating the behaviour of the stereoisomeric hexoses with yeast, the fermentative power of which we now attribute to an enzyme: E. Buchner's zymase.

The experience gained with the glucosides became of service in studying the polysaccharides; for example, maltose and milk sugar were found to differ in their behaviour towards the enzymes of yeast and emulsin much as do $\alpha$ - and $\beta$-methylglucoside. Another outcome of the investigation has been the discovery of distinct enzymes capable of attacking di- and tri-saccharides. Thus lactase was found to be present in Kefir grains and milk sugar yeasts and definite proof was given of the presence of a maltase in beer yeast. As the result of these inquiries, I was able to formulate a rule of general biological significance, namely, that the alcoholic fermentation of a polysaccharide is necessarily preceded by its hydrolysis by some particular enzyme. It was shown, especially in the case of the 
invertase of Monilia Candida, that it is not essential that the enzyme should even be soluble in water, I may mention here that E. Buchner has himself admitted that he was led by these observations on Monilia to make his experiments on the mechanical separation of zymase from the yeast cell.

Unfortunately but few successful syntheses of polysaccharides have been effected. Musculus obtained dextrin-like products from grape sugar by treating it with mineral acids; and I have shown that a disaccharide, isomaltose, may be produced in this way. E. Frankland Armstrong and I have prepared a number of disaccharides by synthesis from the acetylchlorohydroses; one of these products, probably, is identical with melibiose.

But methods such as I refer to are so difficult in their application that their use cannot well be extended to any large number of polysaccharides. It is most desirable therefore that better methods should be devised, as it is probable that the attack on the dextrins, gums and similar undeciphered substances is most likely to be successful if made from the synthetic side. It is to be expected that biology would gain much by the discovery and utilisation of such materials; more, perhaps, than it has from the study of the monosaccharides and of the glucosides prepared by artificial means.

The carbohydrate group is that in which use was first made of enzymes as synthetic agents. You are aware that Croft Hill succeeded in preparing complex carbohydrates by subjecting glucose to the action of the enzymes in beer yeast. His contention that the product is maltose has been controverted; it is more probable that the condensation which glucose undergoes under the influence of the enzyme resembles that effected by hydrogen chloride, that is to say, that the product is a mixture of isomaltose with nonfermentable dextrins. In any case, Croft Hill has the great merit of having been the first to demonstrate the reversibility of enzyme action and since his experiments were made similar results have been obtained with other enzymes, lipase, for example.

An important extension of Croft Hill's discovery is afforded by E. F. Armstrong's observation that the action of emulsin is different from that of the enzymes of yeast, the condensation of glucose taking place in such a way that a disaccharide which appears to be maltose is formed together with other complex products. Such syntheses effected by the agency of enzymes fascinate the imagination, as they approximate closely to natural processes; but I may point out that they cannot take the place of purely chemical methods, as these latter are so much more under our control and can be varied in so many ways that we are in the position to produce materials which it is quite impossible for the organised world to furnish. Labora- 
tory synthetic methods will be indispensable for a long time to come, not only for preparative purposes but also as the means of elucidating the structure of complex substances of natural origin.

This contention is applicable to the proteins even more than it is to the carbohydrates: as they are among the most complex substances produced in the living world and are concerned in all the vital activities of the cell, a complete comprehension of their nature must obviously precede the full development of biological chemistry. I may therefore be allowed to give very briefly a rapid survey of the present position of the chemistry of the proteins. The investigation necessarily began with the isolation and classification of the natural materials; and these were subdivided according to purely external characteristics, such as origin, solubility, coagulability and the like. On the basis of such properties, we distinguish to-day some forty to fifty natural proteins, discovered by the joint labours of chemists and physiologists; but it is to be expected that as the methods of differentiating and separating them are improved, their number will be largely increased.

At present the majority are known only in an amorphous form; some important terms of the group, however, such as oxyhæmoglobin, egg albumin and the albumin of horse serum, excelsin from the Brazil nut and the edestins from other plant seeds have been obtained in definite crystals. But, unfortunately, it cannot be decided from their crystalline appearance whether these products are definite substances, as the tendency to form mixed crystals is the greater the more complicated the molecule. Examples in point are afforded by the aniline dyes, the higher fatty acids and the purine compounds; and those who have studied the chemistry of the natural silicates will be aware of the extension which mineralogists have been compelled to give to the conception of isomorphism. It would therefore be altogether surprising if the crystallised natural proteins should turn out to be single substances. In this gathering of expert chemists I need not dwell on the difficulties to which this uncertainty gives rise.

Of the numerous attempts to unravel the constitution of the proteins by analytical means, the only method which has given useful results hitherto is that of hydrolysis. Hydrolysis can be effected by acids or by alkalis and also by digestive enzymes; the products, it is well known, besides ammonia, are albumoses, peptones and ultimately amino-acids. The wide range of variation in composition of these amino-acids is illustrated in the following table: 
Glycine (Braconnot, 1820).

Alanine (Schiitzenberser, Weyl, 1888).

Valine (v. Gorup-Besanez, 1856).

Leucine (Proust, 1818; Braconnot, 1820). isoLeucine (F. Ehrlich, 1903).

Phenylalanine (E. Schulze and Barbieri, 1881).

Serine (Cramer, 18ñ5).

Tyrosine (Liebig, 1846).

Aspartic acid (Plisson, 1827).

Glutamic acid (Rittbausen, 1866).
Proline (E. Fischer, 1901).

Oxyproline (E. Fischer, 1902).

Ornithine (M. Jaflé, 1877).

Lysine (E. Ireehsel, 1889).

Arginine (E. Schulze and E. Steiger, 1886).

Histidine (A. Kossel, 1896).

Tryptophane (Hopkins and Cole, 1901).

Diaminotrihydroxydodecanoic acid (E. Fischer and E. Abderhalden, Skraup, 1904).

Cystine (Wollaston, 1810 ; K. $\Lambda$. H. Mörner, 1899).

In this table are included all the substances hitherto prepared from the proteins the existence of which is established, with a short reference to their discovery. It may, however, be mentioned that the list is by no means complete; among the new products already announced, the caseanic acid described by Skraup appears to have the greatest claim to recognition as a definite substance. It is certain, however, that the nineteen amino-acids in the table are the chief hydrolytic cleavage products of the proteins and those which are most generally met with. Their discovery has been made during a period of eighty four years, but no less than five of them have been recognised within the present century.

The proportions in which the various amino-acids are obtained from the different proteins vary very considerably. In some cases they are altogether lacking, as may be proved by application of the definite tests for tyrosine, tryptophane or glycine; but it is worthy of note that, as a rule, the amino-acids referred to as isolated from the mixtures produced by subjecting albuminous substances to hydrolysis all occur almost without exception : especially is this true of the important proteins which play the chief part in animal or vegetable metabolism: so that the conclusion must be drawn that none of them can be dispensed with in organic life. With the exception of diaminotrihydroxydodecanoic acid, they have all been so thoroughly investigated that their structure is well established. The majority also have been synthesised, proof of their structure having, in fact, been given in this way. Only oxyproline, histidine and diaminotrihydroxydodecanoic acid remain still to be synthesised.

With the exception of glycine, all the amino-acids derived from natural sources are optically active; but when prepared by ordinary synthetic methods, as is well known, they are obtained in the first instance in the racemic form. The resolution of the racemoids into their optically active components has been effected quite recently in most cases. Asparagine, however, which is closely related to aspartic acid, had been resolved into the two active forms by re- 
crystallising the inactive synthetic product from water and separating the two constituents mechanically. Moreover, in the case of some other amino-acids, for example, leucine, the antipode of the natural form had been obtained by partially fermenting the synthetic product with moulds. The complete synthesis of the active amino-acids which are obtained from natural sources was first accomplished by the method I introduced based upon the use of the acyl derivatives. The method has been applied with success to the majolity of the synthetic products; its extension to the remaining cases, proline, lysine, tryptophane and cystine, is not likely to be atten ded with any difficulties. The synthetical results are summarised in the following table, in which the inactive products are marke. $d l$ and the natural active products are recorded separately:

Glyeme; (Perkin and Duppa, 1858).

Alanine $d l$ - (A. Strecker, 1850).

x- (E. Fischer, 1899).

Valine $d l:$ (Fittig and Clark, 1866).

,,$d$ - (Fischer, 1906).

Leucine $d l .:$ Limpricht; 1855 ; E. Schulze and Likicrnik, 1885).

Leucine $l$ - Fischer, 1900).

isoLeucine $d l$ - (Bouveault and Loquin, 1905).

iscLeuci,ne $d$-(Loquin, 1907).

Pheriylalanine $d l$ - (Erlemmeyer and Lipp, $1($ is3).

Pheny)lalanine $l$ - (Fischer and Scholler, $190 \%$ ).

Serine $\ddot{i} i$ - (Fischer and Leuchs, 1902).

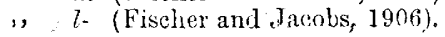

Tyrosine $a l$-(Erlemmeyer and Lipp, 1883). , l- (Fischer, 1900).

Aspartic acid $d l$ - (Dessaignes, 1850). $,, \quad, \quad l$ - (Pintti, I887).

Glutamic acid $d l$-(L. Wolff, 1890).

$,, \quad, \quad d$ - (Eischer, 1899).

Proline dl-(R. Willstiitter, 1900).

Ornithine dl-(Fischer, 1900). , , $d$ - (Sörensen, 1905)

Arginine, activo; partial synthesis from ornithine (E. Schulze and Winterstein, 1899).

Lysine $d l$ - (Fischer and Weigert, 1902).

Tryptophane $d l$ - (A. Ellinger and Flamand, 1907).

Cystine al- (Erlenmeyer, jun., 1903).

i have been able to refer the configuration of some of these subhices to that of glucose. This correlation was first effected thirteen yeai.s ago in the case of aspartic acid. As you are aware, this acid is converted by nitrous acid into $l$-malic acid, the configuration of wl lich can be deduced from its relationship to tartaric acid. In a s.imilar manner, it has been found possible recently to connect serine a nd alanine with active glyceric acid, which Neuberg and Silberinann have been able to correlate with tartaric acid and which it may be anticipated will also be obtained directly from the tetroses by adopting methods such as those introduced by Wohl and Ruff. It will probably be found that the method is applicable to most of the other amino-acids, so that it is to be expected that at no distant date a uniform steric system will be established which will include all the optically active substances in the sugar group, the hydroxyacids and the amino-acids.

It is not without purpose that I have dweit so long on what appear to be side issues of synthetic work, for I firmly believe that 
they will prove in many respects to be of service to biological inquiry. In proof of this, reference may be made to several cases in which they have already borne fruit. For example, the proof given by E. Schulze and Winterstein that arginine is a derivative of guanidine and, therefore, also of urea had undoubtedly some influence in stimulating $\mathrm{H}$. Kossel to the discovery of the interesting manner in which this amino-acid is resolved into urea axid ornithine by means of liver arginase.

To test the assumption that ornithine and lysine are carboxylic acids of tetra- and penta-methylenediamines, Ellinger subjected these substances to the action of putrefactive bacteria and was successful in converting them into the well known basic products of putrefaction.

The manner in which taurine is formed in the animal bocly has been made clear by Friedmann's proof of the relation beitween cystine and taurine.

The isolation of tryptophane by Hopkins and Cole and its conversion into scatole enable us to form a clear picture of the manner in which this offensive substance is produced by htestinal putrefaction and to indicate also the origin of the indoxylsulphuric acid discovered by $\mathrm{E}$. Baumann in urine and of the indigo-blue which is sometimes met with in this secretion. I need do no more than point out, however, that this connexion could not possibly have been established had not $\mathrm{A}$. von Baeyer laid the foundaticin by his great synthetic achievements in the indigo group.

The discovery of isoleucine and its relationship to leucine led F. Ehrlich, a few years ago, to undertake his interesting exp ments on the conversion of amino-acids into primary alcohols by means of beer yeast, which enabled him to give the long-soug i explanation of the formation of fusel oil during alcoholic termentation.

Such instances will be multiplied rapidly in the future; considerations of the relationship of the natural amino-acids to various other classes of substance from the structural and stereochemical point of view are formulated so easily and in such a variety of directions that they can afford much fruitful stimulus to physiological inquiry.

As the amino-acids are formed from the proteins, not only when these are subjected to the action of hot acids and alkalis but also at moderate temperatures by the agency of the digestive enzymes, they are to be regarded as the true foundation stones of protein molecules. Opinions adverse to this hypothesis are only occasionally met with; they centre round the arbitrary supposition that complicated atomic re-arrangements may take place during hydrolysis. 
Were one inclined to regard such objections as of moment, all the experiments on the determination of the constitution of organic compounds by degradation methods would be useless; moreover, the conciusions which have been drawn in other cases from the results obtained by the dissection of compounds have been too frequently confirmed by their synthesis. It is now possible to make this claim on behalf of the proteins, as it has been found to be possible, by a process the reverse of hydrolysis, to associate aminoacids in such a manner that substances are produced which, in the case of the simpler terms, closely resemble peptones, whilst the more complex resemble proteins.

I have termed these synthetic products polypeptides, in view of their relationship to the peptones and to facilitate systematic treatment of the group on the lines followed in the case of the carbohydrates.

No useful purpose will be served by my giving an account of the synthetical methods on the present occasion, especially as I had the honour, six months ago, of describing to you the preparation of an octadecapeptide derived from fifteen molecules of glycine and three molecules of $l$-leucine, a substance which in its external properties closely resembles many natural proteins. I may say that more than one hundred of these artificial polypeptides have already been synthesised.

Many of them, it is true, belong to the lower stages but all the amino-acids previously mentioned, with the exception of diaminotrihydroxydodecanoic acid, have been made use of in their preparation. The synthesis of the higher terms has been restricted hitherto to the combinations of glycine, alanine and leucine; there is not a shadow of doubt, however, that all the remaining aminoacids could be associated in complicated systems with the aid of our present methods. The knowledge of the artificial polypeptides thus acquired has opened up new ways of investigating the peptones and albumoses analytically. During more than fifty years, physiological chemists have endeavoured without much success to isolate homogeneous substances from these ill-defined materials; all the products described by them, however, bear indubitable evidence of being mixtures. By making use of new methods based on the study of the polypeptides, it has been possible during the last two years to isolate and detect with certainty quite a number of dipeptides among the decomposition products of the proteins. In conjunction with Abderhalden, I have succeeded in separating glycyl-d-alanine, $d$-alanyl-l-leucine and $l$-leucyl-d-glutamic acid from silk fibroin, elastin and gliadin respectively. We have definitely proved the existence of dipeptides of glycine with $l$-leucine and $l$-tyrosine in the 
form of anhydrides and our work has made it probable that other combinations exist, for example, that of glycine with valine. P. A. Levene has discovered glycyl-proline anhydride among the products of the digestion of gelatine, whilst T. B. Osborne and S. $\mathbf{H}$. Clapp have noted a dipeptide derived from phenylalanine and proline among the products of the hydrolysis of gliadin by hot sulphuric acid. A tetrapeptide of fairly definite composition has been isolated by Abderhalden and myself from silk fibroin; although its homogeneity is not yet quite established, it is certainly in great part a combination derived from two glycine molecules, one of $d$-alanine and one of $l$-tyrosine. The substance is of special interest on account of its close resemblance to the albumoses. Moreover, l-leucyl-triglycyl-l-tyrosine prepared artificially has all the properties of the albumoses. These observations are of importance as casting doubt on the view which formerly prevailed that, being intermediate products between proteins and peptones, the albumoses are substances of considerable molecular complexity.

This may be true of many of the substances hitherto described under the name, all of which are certainly mixtures; but it is clear that many others are no more complex than the albumose above referred to from silk or the artificial pentapeptide.

In spite of these encouraging successes, I am fully aware of the difficulty of discovering the nature of all the components of the various peptones and albumoses. But in preparing the way for the synthesis of the natural proteins this is not even necessary. Probably the work can be restricted to the reconstruction of the original system from the major products of cleavage formed in the process of hydrolytic dissection. I am indeed venturesome enough to cherish the hope that I may be able to solve this problem in the case of silk fibroin, one of the simplest proteins. To deal with the whole of the proteins will be a gigantic task; so large a number of separate investigations will be necessary that nothing less than the life-work of a whole army of inventive and diligent chemists will suffice to complete it. Probably, too, the unpleasant discovery will be made that the natural proteins as we know them to-day are only to be obtained by mixing the homogeneous artificial products.

I have sketched this prospect merely to indicate the manner in which synthesis must play the leading part in this field of work. The nature of the more complicated carbohydrates, as I have already pointed out, will also have to be determined in the future, I imagine, mainly by the application of synthetic methods. Obviously the conditions are very similar in the case of the dextrins and gums to those met with in the case of the proteins; starch, which has 
hitherto been regarded as a homogeneous substance, appears also to come into the same category, according to Maquenne's observations.

Not only do the proteins constitute the major part of living protoplasm but they appear also to be the material from which the organism prepares its most wonderful agents: the ferments or enzymes. In many of the more thoroughly investigated biological processes their co-operation has been demonstrated and there is good reason to suppose that they take part in all changes occurring within the living cell. So much is certain, the physiological chemistry of the future will be largely concerned with the study of fermentative changes; many indications that this must be the case are to be met with in tracing its latest developments.

The number of the enzymes has been increased to an extraordinary extent during the last ten years. I may allude to the newly-discovered enzymes correlated with the carbohydrates: maltase, lactase, melibiase, trehalase, amygdalase, inulase; to the various oxidases: laccase, tyrosinase; to the lipases, erepsin, enterokinase, arginase, the sucroclastic and glucosidoclastic enzymes and finally to the zymase of alcoholic fermentation. Much valuable information has been accumulated as to the manner in which they act, as to their formation from zymogens and as to their assistance by co-ferments and their retardation by chemical agents or by antiferments. The specific character of their action, in other words, their dependence on the structure and configuration of the object they attack, has been proved beyond doubt and favours very definitely the assumption that enzyme and hydrolyte enter temporarily into combination, a conclusion to which H. E. Armstrong and E. F. Armstrong have quite recently again very properly drawn special attention. But, unfortunately, we know practically nothing of the composition of the enzymes, as the complete isolation of an enzyme has never been accomplished.

From observations hitherto made, it appears in a measure probable that they are derived from proteins and possess a protein-like character. If this be so, it may be hoped that the experience gained with the proteins will be of service in the investigation of enzymes.

In the meantime there are other directions in which synthetic chemistry can be of service in elucidating the chemistry of fermentation. In the same way that the artificial glucosides have been of use in establishing the dependence of the action of enzymes on configuration, the synthesised polypeptides are now being used by Abderhalden, Euler and others to define and measure the activity of the proteoclasts. In a like manner, the synthetic exploration of the 
purine group has served to direct the recent observations on the fermentative de-amination and oxidation of adenine, guanine and xanthine. Finally, attention may be directed to the use that has been made of stereochemical considerations in the course of Bertrand's interesting studies of the oxidation of polyhydric alcohols by the sorbose bacterium.

Not only have the methods of organic chemistry proved to be fruitful of results in the case of the proteins but also when applied to complex derivatives of the latter, such as the nucleo-proteins, for example. Thus we are indebted to the brilliant researches of $\mathrm{A}$. Kossel and his school for our knowledge of no less than four bases of the pyrimidine and purine group obtained by breaking down nucleic acids; the analytical investigation of these latter has already been carried so far that, in the opinion of $\mathrm{H}$. Steudel, it is to be expected that their synthesis will be effected at no distant date. Similar success may be hoped for even sooner in the case of the lecithins. Structural chemistry, moreover, is slowly acquiring the mastery over cholesterin by making use of the experience afforded by the synthetic study of the hydroaromatic substances.

Besides the old well known constituents of the animal body, new substances having quite unexpected properties have been added from time to time. Such are iodothyrin from the thyroid gland-discovered by Baumann - and crystalline adrenaline - isolated by Takamine from the supra-renal capsule-minute doses of which increase the blood-pressure. Judging from analytical results and the synthesis effected by F. Stolz, adrenaline possesses a relatively simple structure. In the opinion of the discoverers, this is probably true of the "pancreatic secretin" made known by Bayliss and Starling's researches, which has the remarkable property of liberating enzymes from the pancreas. May it not also be true of the toxins of many infectious diseases and of the antitoxins used in serum therapeutics, the discovery and systematic investigation of which by Behring, Roux, P. Ehrlich and others are to be reckoned among the greatest achievements of modern biology and medicine?

The methods of organic synthesis will certainly serve to throw light on the nature of all such substances of animal origin. The problems awaiting solution in the plant world are equally numerous.

The great success with which the alkaloids and terpenes have been studied during the past ten years is known to all but it is only too obvious that much still remains to be done when such substances as quinine, morphine and caoutchouc remain to be synthesised.

Alizarin and indigo are prepared artificially in huge quantities and we are well informed as to the structure of hæmatoxylin and 
kindred substances; but our ignorance is correspondingly great of rnost of the blood colouring matters as well as of many coloured constituents of our own bodies: of the hair, the skin and the eye.

The fullest recognition must be accorded, however, to recent investigations on the complex colouring matters of the blood and of chlorophyll, which is distantly related to the former, associated with the names of Schunck, Nencki, Marchlewski, Küster and Willstätter.

In fine, the aid of synthetical chemistry is required in every direction in arriving at a clear understanding of structure and of change. The methods at our disposal in the laboratory are doubtless altogether different from those which come into operation in the living world but chemists are already trying to effect changes in carbon compounds by means of so-called mild interactions, under conditions comparable with those which prevail in the living organism. It may suffice to refer to the development of a number of catalytic processes and to the comprehensive studies on the action of light on organic substances undertaken by Ciamician. In fact, the effort is already being made to co-operate with biology; it is clear that a section of the forces of organic chemistry is being directed once more towards the goal from which it set out. The separation from biology was necessary during the past century while experimental methods and theories were being elaborated; now that our science is provided with a powerful armoury of analytical and synthetical weapons, chemists can once more renew the alliance both to its own honour and to the advantage of biology. Indeed, the prospect of obtaining a clearer insight into the wondrous series of processes which constitute animal and vegetable life may well lead the two sciences to work with definite purpose to a common end.

In order, as far as possible, to avoid mistakes in this difficult task and to shield ourselves from the disappointment which is the inevitable consequence of exaggerated hopes, we cannot do better than strive to imitate the great example of Faraday, who always, with rare acumen, directed his attention to actual phenomena without allowing himself to be influenced by preconceived opinion, and who in his theoretical conceptions gave expression only to observed facts. 